\title{
Evaluation of infectious and non-infectious complications in patients with primary immunodeficiency
}

\author{
SAEED BAZREGARI I,2, GHOLAMREZA AZIZI I,4, MARZIEH TAVAKOL ${ }^{3}$, \\ MOHAMMAD HOSEIN ASGARDOON², FATEMEH KIAEE ${ }^{2}$, NAEIMEH TAVAKOLINIA ${ }^{2}$, \\ AMIR VALIZADEH ${ }^{2}$, HASSAN ABOLHASSANI ${ }^{2,5}$, ASGHAR AGHAMOHAMMADI ${ }^{2}$
}

\begin{abstract}
'Department of Allergy and Immunology, Bandar Abbas Children's Hospital, Hormozgan University of Medical Sciences, Bandar Abbas, Iran ${ }^{2}$ Research Center for Immunodeficiencies, Children's Medical Center, Tehran University of Medical Sciences, Tehran, Iran ${ }^{3}$ Non-Communicable Diseases Research Center, Alborz University of Medical Sciences, Karaj, Iran

${ }^{4}$ Department of Laboratory Medicine, Imam Hassan Mojtaba Hospital, Alborz University of Medical Sciences, Karaj, Iran ${ }^{5}$ Division of Clinical Immunology, Department of Laboratory Medicine, Karolinska Institute at Karolinska University Hospital Huddinge, Stockholm, Sweden
\end{abstract}

\begin{abstract}
Introduction: Primary immunodeficiency diseases (PIDs) are a heterogeneous group of genetic immune disorders. PID patients suffer from a variety of complications. The aim of this study was to determine the infectious and non-infectious complications among PID patients.

Material and methods: This retrospective cohort study was performed on recorded data of 202 PID patients who were diagnosed with eight major categories: common variable immunodeficiency (CVID), X-linked agammaglobulinemia, hyper-IgM syndrome, hyper IgE syndrome, chronic granulomatous disease (CGD), ataxia telangiectasia, hereditary angioedema and leukocyte adhesion deficiency. For all patients, infectious and non-infectious manifestations and laboratory data were collected in a comprehensive questionnaire.

Results: Infectious complications were more frequent than non-infectious complications. Pneumonia and otitis media were the main infectious problems in PID patients, especially in patients with antibody deficiencies. Among the non-infectious complications, splenomegaly and hepatomegaly were the most common complications in PID patients, and were more commonly seen in CGD patients than others. Splenomegaly, hepatomegaly and autoimmunity were the most common findings in CVID patients. A significant correlation was observed between diagnostic delay and bronchiectasis in CVID patients $(p=0.042)$.

Conclusions: PID patients are at risk of multiple infectious and non-infectious problems. Timely diagnosis of PIDs not only improves their outcome and quality of life, but also helps prevent these troubling complications.
\end{abstract}

Key words: primary immunodeficiency, infection, pneumonia, autoimmunity.

(Centr Eur J Immunol 2017; 42 (4): 336-341)

\section{Introduction}

Primary immunodeficiency diseases (PIDs) are a heterogeneous group of immune disorders that affect distinct components of the humeral and cellular arms of the immune system. PIDs are caused by hereditary or genetic defects, and, although some disorders present at birth or in early childhood, the disorders can affect everybody, regardless of age or gender [1-3].

It is known that PID patients suffer from a variety of clinical complications including infectious and non-infec- tious diseases [4, 5]. PID patients are mainly characterized by increased susceptibility to infections because there are defects in neutrophils, macrophages, complement proteins and lymphocyte subsets $[1,6,7]$. Infections involving multiple organs, such as the respiratory tract, gastrointestinal tract (chronic diarrhea and enteropathy), central nervous system (meningitis and encephalitis), skin (cellulitis, impetigo and recurrent abscesses), and septicemia, are more prevalent in PID patients [8]. Among the infectious complications, respiratory signs and symptoms present a significant cause of mortality and morbidity among patients

Correspondence: Prof. Asghar Aghamohammadi, Children's Medical Center Hospital, 62 Qarib St., Keshavarz Blvd., Tehran 14194, Iran, tel. + 98216642 8998, fax + 98216692 3054, e-mail: aghamohammadi@tums.ac.ir

Submitted: 30.06.2016; Accepted: 22.08.2016 
suffering from PIDs and they are observed in both children and adults. These complications can affect primarily either the upper respiratory tract (e.g., sinusitis and otitis media) or lower respiratory tract (e.g., pneumonia, bronchiectasis, and interstitial lung diseases). The lower respiratory tract complications are usually considered to be more important and also more specific for PID patients' prognosis [9, 10].

PID patients may also present with a variety of non-infectious complications including malignant, allergic, inflammatory, and autoimmune manifestations $[11,12]$. In some cases, these non-infectious manifestations (such as autoimmune diseases) may be the first or the predominant clinical manifestation of the underlying immunodeficiency [13]. Therefore, early diagnosis and adequate therapy are the key facts for survival and a better quality of life in PID patients, while the delay in diagnosis and inadequate management of these disorders may lead to permanent organ damage $[14,15]$.

This study aimed to evaluate the clinical findings of infectious and non-infectious complications in eight main types of PIDs: common variable immunodeficiency (CVID), X-linked agammaglobulinemia (XLA), hyper-IgM syndrome (HIgM), hyper-IgE syndrome (HIES), chronic granulomatous disease (CGD), ataxia telangiectasia (AT), hereditary angioedema (HAE) and leukocyte adhesion deficiency (LAD).

\section{Material and methods}

\section{Patients}

This retrospective cohort study was performed on patients with PIDs who were diagnosed and treated in the Research Center for Immunodeficiencies at the Children's Medical Center affiliated to Tehran University of Medical Sciences. The study was reviewed and approved by the Ethics Committee of the Tehran University of Medical Sciences. PID patients were diagnosed based on the Pan-American Group for Immunodeficiency (PAGID) and the European Society for Immunodeficiency (ESID) diagnostic criteria [16]. Patients then were registered in the national database [8] and then classified into different categories of PIDs. A selected group of patients with high incidence ( $n>10$ patients) were enrolled in this study. Therefore, available patients with diagnosis of CVID, XLA, HIgM, HIES, CGD, AT, HAE and LAD were selected. Severe combined immunodeficiency patients were not included because of the bias of heterogeneity of clinical condition and follow-up times depend on the availability of hematopoietic stem cell transplantation. We designed a comprehensive questionnaire that included demographic information including name, age at clinical presentation, age at diagnosis at our clinic and age at last follow-up, diagnosis, family history of PID, infections, associated autoimmune disease and allergy, enteropathy, lymphoprolifer- ative and malignancies. The questionnaire was completed for all patients. Moreover, laboratory test data including the initial immunological work-up at the time of referral to our research center were also recorded for each patient.

\section{Statistical analyses}

Statistical analyses were performed using the SPSS software package, version 22 (SPSS Inc., Chicago, IL, USA). To report the results, values were expressed as frequency (number and percentage), mean \pm SD and median (IQR), as appropriate. Fisher's exact test and the chisquare test were used for comparison of categorical variables, whereas the t-test and one-way ANOVA were used to compare numerical variables. Pearson's and Spearman correlation coefficient were calculated for assessment of correlations between quantitative and qualitative variables, respectively. A $p$-value $<0.05$ was considered significant.

\section{Results}

\section{Demographic and characteristic baseline}

In this retrospective study, 202 PID patients (128 males and 74 females) with antibody deficiency (XLA, CVID, HIgM; $n=91$ ), congenital defects of phagocyte (CGD and LAD; $n=32$ ), complement deficiency (HAE; $n=8$ ), and combined immunodeficiencies (AT and HIES; $n=71$ ) were evaluated. Demographic and corresponding immunologic data for these eight types of PIDs are presented in Table 1. The median age of these PID patients was 14 (3.0-40.0) years. The median diagnostic delay was found to be $31.0(6.0-264.0)$ months, while the longest and shortest delay in diagnosis was observed in patients with HAE and LAD, respectively. There was a remarkable difference in the duration of diagnostic delay between patients with CVID (46.5 months) compared to XLA (24.0 months, $p=0.043), \operatorname{HIgM}$ (18.0 months, $p=0.078)$, CGD (7.5 months, $p=0.035)$ and LAD (3.5 months, $p=0.002)$. A significant correlation was observed between diagnostic delay and bronchiectasis in CVID patients $(r=0.323, p=0.042)$. However, no significant correlation was found between delay in diagnosis and chronic diarrhea in patients with CVID, although this association was borderline significant in XLA patients ( $r=0.354, p=0.055$ ). Parental consanguinity was observed in $50.4 \%$ of cases. Among them, LAD patients had the highest rate $(100 \%$ of cases) while XLA patients had the lowest (36.7\% of cases).

\section{Infectious complications}

Infectious complications were more frequent than non-infectious complications in PID patients. Pneumonia $(69.3 \%)$ and otitis media (56.9\%) were the main infectious problems in PID patients, especially in patients with antibody deficiencies. A detailed report of infectious complications in eight different groups is summarized in Table 2. 
Table 1. Demographic and corresponding immunologic data for PID patients

\begin{tabular}{lccccccccc}
\hline Factor & $\begin{array}{c}\text { Total } \\
(\boldsymbol{N}=\mathbf{2 0 2})\end{array}$ & $\begin{array}{c}\text { XLA } \\
(\boldsymbol{n}=\mathbf{3 0})\end{array}$ & $\begin{array}{c}\text { CVID } \\
(\boldsymbol{n}=\mathbf{4 0})\end{array}$ & $\begin{array}{c}\text { HIgM } \\
(\boldsymbol{n}=\mathbf{2 1})\end{array}$ & $\begin{array}{c}\text { CGD } \\
(\boldsymbol{n}=\mathbf{2 0})\end{array}$ & $\begin{array}{c}\text { LAD } \\
(\boldsymbol{n}=\mathbf{1 2})\end{array}$ & $\begin{array}{c}\text { AT } \\
(\boldsymbol{n}=\mathbf{2 0})\end{array}$ & $\begin{array}{c}\text { HIES } \\
(\boldsymbol{n}=\mathbf{5 1})\end{array}$ & $\begin{array}{c}\text { HAE } \\
(\boldsymbol{n}=\mathbf{8})\end{array}$ \\
\hline Sex ratio (M/F) & $128 / 74$ & $30 / 0$ & $25 / 15$ & $17 / 4$ & $13 / 7$ & $6 / 6$ & $10 / 10$ & $25 / 26$ & $2 / 6$ \\
\hline Age (years) & 14.0 & 16.0 & 16.0 & 9.0 & 9.0 & 9.5 & 10.0 & 14.0 & 23.5 \\
& $(3.0-40.0)$ & $(7.7-25.5)$ & $(12.0-24.5)$ & $(3.0-12.0)$ & $(6.5-18.7)$ & $(3.7-13.0)$ & $(8.25-18.5)$ & $(3.0-22.0)$ & $(3.1-40.5)$ \\
\hline $\begin{array}{l}\text { Delay diagnosis } \\
\text { (months) }\end{array}$ & 31.0 & 24.0 & 46.5 & 18.0 & 7.5 & 3.5 & 44.0 & 84.0 & 60.4 \\
\hline Consanguinity, & $102(5-264.0)$ & $(5.5-40.5)$ & $(19.0-81.5)$ & $(2.5-48.0)$ & $(2.2-19.5)$ & $(1.2-14.5)$ & $(13.5-60.0)$ & $(6.1-191.0)$ & $(1.3-264.0)$ \\
$n$ (\%) & $11(36.7)$ & $25(62.5)$ & $13(61.9)$ & $13(65.0)$ & $12(100.0)$ & $17(85.0)$ & $10(19.6)$ & $1(12.5)$ \\
\hline IgG (mg/dl) & 250 & 100 & 242 & 70 & 1572 & 781 & 700 & 1396 & 1420 \\
& $(42-607)$ & $(18-290)$ & $(64-334)$ & $(14-129)$ & $(960-1935)$ & $(616-1800)$ & $(550-1093)$ & $(740-2010)$ & $(941-2010)$ \\
\hline IgA (mg/dl) & 9 & 5 & 7 & 8 & 182 & 131 & 36 & 200 & 140 \\
& $(3-38)$ & $(0-20)$ & $(2-22)$ & $(5-20)$ & $(95-414)$ & $(89-378)$ & $(5-116)$ & $(150-340)$ & $(105-225)$ \\
\hline IgM (mg/dl) & 49 & 5 & 17 & 271 & 153 & 146 & 224 & 140 & 230 \\
& $(10-186)$ & $(0-17)$ & $(6-35)$ & $(115-1308)$ & $(128-218)$ & $(87-194)$ & $(126-313)$ & $(58-230)$ & $(68-345)$ \\
\hline IgE (IU/ml) & 2.5 & 4 & 1 & 1 & 105 & - & 4 & 9040 & 54 \\
& $(1-11)$ & $(1-6)$ & $(0-3)$ & $(0-3)$ & $(15-792)$ & & $(1-10)$ & $(1700-15720)$ & $(10-118)$ \\
\hline
\end{tabular}

Ig-immunoglobulins; XLA-X-linked agammaglobulinemia; CVID - common variable immune deficiency; HIgM-hyper-IgM syndrome; HIES - hyper-IgE syndrome; HAE - hereditary angioedema; CGD - chronic granulomatous disease; AT - ataxia-telangiectasia; LAD - leukocyte adhesion deficiency

Note. For age, delay diagnosis and Ig levels the median is shown with $25^{\text {th }}$ and $75^{\text {th }}$ percentiles

Table 2. Infectious complications among PID patients

\begin{tabular}{lccccccccc}
\hline $\begin{array}{l}\text { Infectious } \\
\text { complications }\end{array}$ & $\begin{array}{c}\text { Total } \\
(\boldsymbol{N}=\mathbf{2 0 2})\end{array}$ & $\begin{array}{c}\text { XLA } \\
(\boldsymbol{n}=\mathbf{3 0})\end{array}$ & $\begin{array}{c}\text { CVID } \\
(\boldsymbol{n}=\mathbf{4 0})\end{array}$ & $\begin{array}{c}\text { HIgM } \\
(\boldsymbol{n}=\mathbf{2 1})\end{array}$ & $\begin{array}{c}\text { CGD } \\
(\boldsymbol{n}=\mathbf{2 0})\end{array}$ & $\begin{array}{c}\text { LAD } \\
(\boldsymbol{n}=\mathbf{1 2})\end{array}$ & $\begin{array}{c}\text { AT } \\
(\boldsymbol{n}=\mathbf{2 0})\end{array}$ & $\begin{array}{c}\text { HIES } \\
(\boldsymbol{n}=\mathbf{5 1})\end{array}$ & $\begin{array}{c}\text { HAE } \\
(\boldsymbol{n}=\mathbf{8})\end{array}$ \\
\hline Otitis media & $115(56.9)$ & $19(63.3)$ & $27(67.5)$ & $12(57.1)$ & $4(20.0)$ & $2(16.7)$ & $10(50.0)$ & $40(78.5)$ & $1(12.5)$ \\
\hline Pneumonia & $140(69.3)$ & $20(66.7)$ & $33(82.5)$ & $16(76.2)$ & $15(75.0)$ & $3(25.0)$ & $13(65.0)$ & $40(78.5)$ & $0(0.0)$ \\
\hline Chronic cough & $86(42.5)$ & $17(56.7)$ & $26(65.0)$ & $8(38.1)$ & $1(5.0)$ & $0(0.0)$ & $3(15.0)$ & $30(58.8)$ & $1(12.5)$ \\
\hline Abscess & $78(38.6)$ & $4(13.3)$ & $5(12.5)$ & $3(14.3)$ & $20(100.0)$ & $5(41.7)$ & $2(10.0)$ & $39(76.4)$ & $0(0.0)$ \\
\hline Osteomyelitis & $7(3.4)$ & $1(3.3)$ & $0(0.0)$ & $0(0.0)$ & $6(30.0)$ & $0(0.0)$ & $0(0.0)$ & $0(0.0)$ & $0(0.0)$ \\
\hline Meningitis & $10(4.9)$ & $6(20.0)$ & $4(10.0)$ & $0(0.0)$ & $0(0.0)$ & $0(0.0)$ & $0(0.0)$ & $0(0.0)$ & $0(0.0)$ \\
\hline Septic arthritis & $23(11.3)$ & $6(20.1)$ & $7(17.5)$ & $4(19.0)$ & $4(20.0)$ & $2(16.5)$ & $0(0.0)$ & $0(0.0)$ & $0(0.0)$ \\
\hline Chronic diarrhea & $47(23.2)$ & $8(26.7)$ & $19(47.5)$ & $5(23.8)$ & $7(35.0)$ & $4(33.3)$ & $4(20.0)$ & $0(0.0)$ & $0(0.0)$ \\
\hline Skin ulcers & $64(31.6)$ & $0(0.0)$ & $1(2.5)$ & $0(0.0)$ & $11(55.5)$ & $10(83.3)$ & $0(0.0)$ & $41(80.3)$ & $1(12.5)$ \\
\hline Omphalitis & $8(3.9)$ & $0(0.0)$ & $0(0.0)$ & $0(0.0)$ & $0(0.0)$ & $8(66.7)$ & $0(0.0)$ & $0(0.0)$ & $0(0.0)$ \\
\hline Mouth sores & $37(18.3)$ & $0(0.0)$ & $2(5.0)$ & $2(9.5)$ & $2(10.0)$ & $5(41.7)$ & $3(15)$ & $23(45.1)$ & $0(0.0)$ \\
\hline Bronchiectasis & $39(37.9)$ & $5(16.7)$ & $20(50.0)$ & $5(23.8)$ & $1(5.0)$ & $0(0.0)$ & $0(0.0)$ & $8(15.7)$ & $0(0.0)$ \\
\hline
\end{tabular}

XLA - X-linked agammaglobulinemia; CVID - common variable immune deficiency; HIES - hyper-IgE syndrome; HAE - hereditary angioedema; HIgM - hyperIgM syndrome; CGD - chronic granulomatous disease; $A T$ - ataxia-telangiectasia; LAD - leukocyte adhesion deficiency

The most frequent infectious complications in XLA and HIgM patients were pneumonia $(66.7 \%$ and $76.2 \%$, respectively) and otitis media (63.3\% and $57.1 \%$, respectively). However, pneumonia (82.5\%) and otitis media (67.5\%) were more common in CVID than other disorders. There were diverse infectious manifestations in CVID patients; in addition to pneumonia and otitis media, chronic cough $(65.0 \%)$, bronchiectasis $(50.0 \%)$, and chronic diarrhea
(47.5\%) were also frequently documented in these patients. In CGD patients, abscess (100.0\%), pneumonia $(75.0 \%)$, skin ulcers $(55.5 \%)$, and osteomyelitis $(30.0 \%)$ were seen more, while in LAD patients, skin ulcers $(83.3 \%)$, omphalitis $(66.7 \%)$, abscess $(41.7 \%)$ and mouth sores $(41.7 \%)$ were the most common findings. Similar to antibodydeficient patients, pneumonia $(65.0 \%)$ and otitis media $(50.0 \%)$ were the most common infectious manifestations 
in AT patients. Bronchiectasis was seen in $33 \%$ of PAD patients and was significantly more common than other PIDs $(p<0.001)$. There was significantly higher prevalence of bronchiectasis in CVID in comparison with XLA $(p=0.004)$ and $\operatorname{HIgM}(p=0.048)$. Likewise, a higher rate of chronic diarrhea was observed in CVID patients than $\operatorname{XLA}(p=0.076), \operatorname{HIgM}(p=0.072)$ and $\operatorname{AT}(p=0.039)$. Interestingly, septicemia was not reported in any of these PID patients.

\section{Non-infectious complications}

In this study, 22 PID patients (10.9\%) had only non-infectious manifestations (2 CVID, 1 XLA, 1 HIgM, 1 AT, 10 HIES and 7 HAE patients). Among the non-infectious complications in PID patients, eczema (28.7\%), clubbing $(15.8 \%)$, splenomegaly $(14.3 \%)$, and hepatomegaly $(9.9 \%)$ were the most common. Non-infectious complications in the study groups are summarized in Table 3. Splenomegaly $(40 \%)$ and hepatomegaly (30\%) were more common in CGD patients than others. The most common complications in XLA patients were JRA (13.3\%), eczema (6.7\%), and hepatomegaly $(6.7 \%)$, while in HIgM patients splenomegaly $(14.3 \%)$, hepatomegaly $(14.3 \%)$, and neutropenia $(23.8 \%)$ were more prevalent. Splenomegaly (35.0\%), hepatomegaly $(15.0 \%)$ and autoimmunity including AIHA $(15.0 \%)$, ITP $(12.5 \%)$ and JRA $(17.5 \%)$ were the most common findings in CVID patients. Malignancy was only reported in two (5\%) patients with CVID and one (1.9\%) with HIES. As described above, CVID was most commonly associated with autoimmunity. There was a significantly higher prevalence of autoimmunity manifestations in
CVID compared to XLA $(p=0.004), \operatorname{HIgM}(p=0.009)$, $\operatorname{CGD}(p=0.011)$, AT $(p=0.011)$ and $\operatorname{LAD}(p=0.023)$.

\section{Discussion}

PIDs are a heterogeneous group of genetic immune disorders. The number of these disorders is increasing annually and over 270 PIDs are defined so far [17]. PIDs are principally manifested by vulnerability to recurrent infection [13]. However, there are multiple noninfectious features accompanied by these conditions $[11,18]$.

This is a report of infectious and noninfectious manifestations in a group of PIDs including patients with antibody deficiency (XLA, HIgM and CVID), phagocytic disorders (CGD and LAD) and combined immunodeficiency (AT and HIES) and HAE. To the best of our knowledge, this is the first survey on the infectious and non-infectious complications of different types of PID.

Most of the PIDs present with regular infections in upper and lower respiratory tracts that physicians usually do not take any notice of except for having a strong suspicion and doing an early diagnostic workup; otherwise these conditions will be diagnosed with a significant delay [13]. Diagnostic delay (mean: 24 months) was an important finding in this survey. Patients with CVID and AT had the longest mean duration (46.5 months and 44 months) of delay in diagnosis whereas LAD was diagnosed at the earliest time (3.5 months). This discrepancy could be explained by the severity and features of the diseases. The first manifestation of LAD is delayed separation of the umbilical cord and cutaneous ulcers [18]. Therefore, it

Table 3. Non-infectious complications among PID patients

\begin{tabular}{|c|c|c|c|c|c|c|c|c|c|}
\hline $\begin{array}{l}\text { Non-infectious } \\
\text { complications }\end{array}$ & $\begin{array}{c}\text { Total } \\
(N=\mathbf{2 0 2})\end{array}$ & $\begin{array}{c}\text { XLA } \\
(n=30)\end{array}$ & $\begin{array}{c}\text { CVID } \\
(n=40)\end{array}$ & $\begin{array}{c}\text { HIgM } \\
(n=21)\end{array}$ & $\begin{array}{c}\text { CGD } \\
(n=20)\end{array}$ & $\begin{array}{c}\text { AT } \\
(n=20)\end{array}$ & $\begin{array}{c}\text { LAD } \\
(n=12)\end{array}$ & $\begin{array}{c}\text { HIES } \\
(n=51)\end{array}$ & $\begin{array}{c}\text { HAE } \\
(n=8)\end{array}$ \\
\hline Splenomegaly & $29(14.3)$ & $1(3.3)$ & $14(35.0)$ & $3(14.3)$ & $8(40.0)$ & $1(5.0)$ & $2(16.7)$ & $0(0.0)$ & $0(0.0)$ \\
\hline Hepatomegaly & $20(9.9)$ & $2(6.7)$ & $6(15.0)$ & $3(14.3)$ & $6(30.0)$ & $1(5.0)$ & $2(16.7)$ & $0(0.0)$ & $0(0.0)$ \\
\hline JRA & $12(5.9)$ & $4(13.3)$ & $7(17.5)$ & $1(4.8)$ & $0(0.0)$ & $0(0.0)$ & $0(0.0)$ & $0(0.0)$ & $0(0.0)$ \\
\hline AIHA & $7(3.4)$ & $1(3.3)$ & $6(15.0)$ & $0(0.0)$ & $0(0.0)$ & $0(0.0)$ & $0(0.0)$ & $0(0.0)$ & $0(0.0)$ \\
\hline ITP & $8(3.9)$ & $1(3.3)$ & $5(12.5)$ & $0(0.0)$ & $1(5.0)$ & $1(5.0)$ & $0(0.0)$ & $0(0.0)$ & $0(0.0)$ \\
\hline Neutropenia & $9(4.4)$ & $3(10.0)$ & $0(0.0)$ & $5(23.8)$ & $0(0.0)$ & $0(0.0)$ & $0(0.0)$ & $1(1.9)$ & $1(12.5)$ \\
\hline Hypothyroidism & $2(0.9)$ & $0(0.0)$ & $2(5.0)$ & $0(0.0)$ & $0(0.0)$ & $0(0.0)$ & $0(0.0)$ & $0(0.0)$ & $0(0.0)$ \\
\hline Diabetes & $1(0.4)$ & $0(0.0)$ & $0(0.0)$ & $1(4.8)$ & $0(0.0)$ & $0(0.0)$ & $0(0.0)$ & $0(0.0)$ & $0(0.0)$ \\
\hline Vitiligo & $1(0.4)$ & $0(0.0)$ & $1(2.5)$ & $0(0.0)$ & $0(0.0)$ & $0(0.0)$ & $0(0.0)$ & $0(0.0)$ & $0(0.0)$ \\
\hline Alopecia & $3(1.4)$ & $1(3.3)$ & $2(5.0)$ & $0(0.0)$ & $0(0.0)$ & $0(0.0)$ & $0(0.0)$ & $0(0.0)$ & $0(0.0)$ \\
\hline Eczema & $58(28.7)$ & $2(6.7)$ & $1(2.5)$ & $0(0.0)$ & $0(0.0)$ & $2(10.0)$ & $0(0.0)$ & $48(94.1)$ & $5(62.5)$ \\
\hline Clubbing & $32(15.8)$ & $4(13.3)$ & $16(40)$ & $5(23.8)$ & $3(15)$ & $1(5)$ & $0(0.0)$ & $3(5.8)$ & $0(0.0)$ \\
\hline Paralysis & $2(0.9)$ & $2(6.6)$ & $0(0.0)$ & $0(0.0)$ & $0(0.0)$ & $0(0.0)$ & $0(0.0)$ & $0(0.0)$ & $0(0.0)$ \\
\hline Malignancy & $3(1.4)$ & $0(0.0)$ & $2(5.0)$ & $0(0.0)$ & $0(0.0)$ & $0(0.0)$ & $0(0.0)$ & $1(1.9)$ & $0(0.0)$ \\
\hline
\end{tabular}


is predictable that LAD would be diagnosed much earlier than CVID, XLA and AT with the vague presentations in the early months of life. Consanguinity of the parents was frequently found in the patients. All of our LAD patients had related parents, but this variable was found to be positive in about a third of patients with XLA. This could be explained by the pattern of inheritance that is autosomal recessive in the LAD and X-linked in XLA.

The respiratory system is substantially the most prominent target organ for both infectious and noninfectious complications of PIDs, and primary antibody deficiencies are the leading cause of these complications [9]. Regarding the infectious complications, pneumonia was generally the most common problem in our patients, and CVID was mainly associated with pneumonia while LAD was least often accompanied by pneumonia. HIgM, CGD, XLA and AT were also associated with pneumonia, in descending order. The lung is not the first target in LAD, and involvement of superficial tissues including skin and mucus membranes is more predominant in this entity. This could also be explained by the younger age of the LAD patients at the time of diagnosis compared to the CVID patients with the longest delay of diagnosis. Sinusitis and otitis media were found to be the most common infections in CVID patients with long-term diagnostic delay, while chronic diarrhea (36\%) was the most common infection in CVID patients with shorter duration of diagnostic delay in the previous study by Aghamohammadi et al. They also found that pneumonia was significantly more frequent in the CVID patients with a long duration of delay in diagnosis [19]. Oksenhendler et al. evaluated the infections in 252 CVID patients; $91 \%$ of their patients had respiratory problems, bronchitis $(69 \%)$, sinusitis $(63 \%)$ and pneumonia (58\%) being the most common infections [20].

Bronchiectasis, which generally is a complication of recurrent uninhibited pulmonary infections, is considered as a clue for the diagnosis of PIDs [9]. A significant increase in bronchiectasis occurrence has been reported in a group of 47 CVID patients with delayed diagnosis [19]. We found bronchiectasis in half of the patients with CVID, and its occurrence in patients with HIgM and XLA was lower, while none of the patients with AT and LAD had bronchiectasis. This might be explained by the more frequent pneumonia in CVID, HIgM and XLA. In addition, the older age of the CVID and XLA patients in our survey compared to HIgM and CGD could be an explanation for the difference between these conditions. Our findings are comparable with previous studies on the infectious complications in CVID [19-21]. The younger age at the diagnosis of LAD patients and the lower propensity for lung involvement in this disease could be the reason for the lack of bronchiectasis in this group. Aghamohammadi et al. found bronchiectasis as the principle complication in their study group, and it was more significantly increased in the CVID patients with long duration of delay diagnosis
(58\% in long lasting diagnostic delay compared to $34 \%$ in the short diagnostic delay) [19]. Habahbeh et al. found pneumonia $(62.3 \%)$, otitis media $(49.1 \%)$ and sinusitis $(34 \%)$ as the most common infections in 53 patients with primary antibody deficiency [21].

Pulmonary infections are usually observed in AT patients [18]. Although bronchiectasis is expected to be a manifestation of this disease, we did not find this complication in our patients. It needs to be studied in future to find other probable risk factors such as genetic characteristics for bronchiectasis other than recurrent infection in PID.

Recurrent otitis media (4 or more episode of ear infection in a year) is considered one of the warning signs of PIDs [9]. Otitis media was observed in more than half $(56.9 \%)$ of our study group. CVID (67.5\%), XLA (63.3\%), HIgM (57.1\%) and AT (50\%) were more associated with otitis media, while otitis media frequency in LAD (16.7\%) and CGD (20\%) was lower. This observation is due to the importance of humoral immunity in the defense against the encapsulated bacteria that are the leading cause of otitis media.

Skin and mucosal manifestations including ulcer and abscess formation are frequently considered the common features of phagocytic disorders [13]. Skin ulcer and abscess were found in all of the CGD patients in this study. However, LAD patients presented with omphalitis $(66.7 \%)$, mouth sores $(41.7 \%)$ and skin ulcer $(83.3 \%)$.

According to the non-infectious complications, splenomegaly, clubbing and hepatomegaly were more common than others. CVID was the most predisposing immunodeficiency for these signs. Chronic infection and immune dysregulation could explain the higher frequency of the hepatosplenomegaly in CVID [22]. Clubbing is frequently considered a common feature of chronic lung infections and bronchiectasis [23]. Therefore, the higher prevalence of a chronic cough, pneumonia and bronchiectasis in our patients with CVID could be the reason for more frequent clubbing in these patients compared to other groups.

CVID is identified as an immune dysregulation entity that is commonly associated with different features of autoimmunity involving hematologic, cutaneous, endocrinologic, gastrointestinal and rheumatologic systems [22, 24-26]. We found autoimmune disorders (JRA, ITP, and AIHA) in patients with CVID more frequent than other study groups. Cytopenias were commonly seen in the CVID group including AIHA (15\%) and ITP $(12.5 \%)$ in addition to neutropenia in XLA (10\%) and HIgM (23.8\%) in the present study. Autoimmune cytopenias (22\%) were the most common autoimmunity in primary antibody deficiencies in the Habahbeh et al. survey. They found these cytopenias in $50 \%$ of the CVID group [21]. Oksenhendler et al. also found autoimmune cytopenias in $18 \%$ of CVID patients [20]. Wang and coworkers found a frequency of autoimmune cytopenias (AIHA and ITP) of $11 \%$ in 326 CVID patients [27]. These are similar to our finding that CVID was more commonly associated with AIHA and ITP. 
Other non-infectious manifestations of immunodeficiency including skin disorders (alopecia, vitiligo and eczema), endocrine disorders (hypothyroidism and diabetes), paralysis and malignancy were found to be uncommon, and CVID was the only predisposing PID for most of them.

\section{Conclusions}

The results of our study showed that PID patients are at risk of multiple infectious and non-infectious problems. Timely diagnosis of PIDs not only improves their outcome and quality of life, but also helps to prevent these troubling complications. Recurrent, severe infections and autoimmune disorders could be the first manifestation of PIDs. Considering PID in the differential diagnosis of these problems leads to minimizing the delay in diagnosis and improvement the prognosis in PIDs. More detailed studies with more patients and a broader list of studied PIDs are required to find the best way and time of diagnosis and appropriate preventive measures.

\section{The authors declare no conflict of interest.}

\section{References}

1. Geha RS, Notarangelo LD, Casanova JL, et al. (2007): Primary immunodeficiency diseases: an update from the International Union of Immunological Societies Primary Immunodeficiency Diseases Classification Committee. J Allergy Clin Immunol 120: 776-794.

2. Mohammadinejad P, Pourhamdi S, Abolhassani H, et al. (2015): Primary Antibody Deficiency in a Tertiary Referral Hospital: A 30-Year Experiment. J Invest Allergol Clin Immunol 25: 416-425.

3. Nabavi M, Arshi S, Bemanian MH, et al. (2016): Long-term follow-up of ninety eight Iranian patients with primary immune deficiency in a single tertiary centre. Allergol Immunopathol 44: 322-330.

4. Takada H (2013): Primary immunodeficiency in Japan; epidemiology, diagnosis, and pathogenesis. Pediatr Int 55: 671674.

5. Aghamohammadi A, Mohammadinejad P, Abolhassani H, et al. (2014): Primary immunodeficiency disorders in Iran: update and new insights from the third report of the national registry. J Clin Immunol 34: 478-490.

6. Sharifi L, Mirshafiey A, Rezaei N, et al. (2016): The role of toll-like receptors in B-cell development and immunopathogenesis of common variable immunodeficiency. Expert Rev Clin Immunol 12: 195-207.

7. Yazdani R, Fatholahi M, Ganjalikhani-Hakemi M, et al. (2016): Role of apoptosis in common variable immunodeficiency and selective immunoglobulin A deficiency. Mol Immunol 71: 1-9.

8. Aghamohammadi A, Moghaddam ZG, Abolhassani H, et al. (2014): Investigation of underlying primary immunodeficiencies in patients with severe atopic dermatitis. Allergol Immunopathol 42: 336-341.
9. Jesenak M, Banovcin P, Jesenakova B, Babusikova E (2014): Pulmonary manifestations of primary immunodeficiency disorders in children. Front Pediatr 2: 77.

10. Aghamohammadi A, Abolhassani H, Mohammadinejad P, Rezaei N (2012): The approach to children with recurrent infections. Iran J Allergy Asthma Immunol 11: 89-109.

11. Kumar A, Teuber SS, Gershwin ME (2006): Current perspectives on primary immunodeficiency diseases. Clin Develop Immunol 13: 223-259.

12. Bruton OC (1952): Agammaglobulinemia. Pediatrics 9: 722-728.

13. McCusker C, Warrington R (2011): Primary immunodeficiency. Allergy Asthma Clin Immunol 7 Suppl 1: S11.

14. Brodszki N, Jonsson G, Skattum L, Truedsson L (2014): Primary immunodeficiency in infection-prone children in southern Sweden: occurrence, clinical characteristics and immunological findings. BMC Immunol 15: 31.

15. Azizi G, Abolhassani H, Asgardoon MH, et al. (2016): Managing patients with side effects and adverse events to immunoglobulin therapy. Expert Rev Clin Pharmacol 9: 91-102.

16. Conley ME, Notarangelo LD, Etzioni A (1999): Diagnostic criteria for primary immunodeficiencies. Representing PAGID (Pan-American Group for Immunodeficiency) and ESID (European Society for Immunodeficiencies). Clin Immunol 93: 190-197.

17. Picard C, Al-Herz W, Bousfiha A, et al. (2015): Primary Immunodeficiency Diseases: an Update on the Classification from the International Union of Immunological Societies Expert Committee for Primary Immunodeficiency 2015. J Clin Immunol 35: 696-726.

18. Al-Herz W, Bousfiha A, Casanova JL, et al. (2014): Primary immunodeficiency diseases: an update on the classification from the international union of immunological societies expert committee for primary immunodeficiency. Front Immunol 5: 162.

19. Aghamohammadi A, Tavassoli M, Abolhassani H, et al. (2009): Infectious and non-infectious complications among undiagnosed patients with common variable immunodeficiency. Iran J Pediatr 19: 367-375.

20. Oksenhendler E, Gérard L, Fieschi C, et al. (2008): Infections in 252 patients with common variable immunodeficiency. Clin Infect Dis 46: 1547-1554.

21. Habahbeh ZM, Abu-Shukair ME, Almutereen MA, et al. (2014): Primary Antibody Deficiencies at Queen Rania Children Hospital in Jordan: Single Center Experience. Iran J Immunol 11: 49.

22. Agarwal S, Cunningham-Rundles C (2009): Autoimmunity in common variable immunodeficiency. Curr Allergy Asthma Reports 9: 347-352.

23. Dickinson C (1993): The aetiology of clubbing and hypertrophic osteoarthropathy. Eur J Clin Invest 23: 330-338.

24. Cunningham-Rundles $C$ (2008): Autoimmune manifestations in common variable immunodeficiency. J Clin Immunol 28: 42-45.

25. Boileau J, Mouillot G, Gérard L, et al. (2011): Autoimmunity in common variable immunodeficiency: correlation with lymphocyte phenotype in the French DEFI study. J Autoimmun 36: $25-32$.

26. Michel M, Chanet V, Galicier L, et al. (2004): Autoimmune thrombocytopenic purpura and common variable immunodeficiency: analysis of 21 cases and review of the literature. Medicine 83: 254-263.

27. Wang J, Cunningham-Rundles C (2005): Treatment and outcome of autoimmune hematologic disease in common variable immunodeficiency (CVID). J Autoimmun 25: 57-62. 